\title{
Some phenomenologies of a simple scotogenic inverse seesaw model
}

\author{
Yi-Lei Tang ${ }^{*}$ \\ School of Physics, KIAS, 85 Hoegiro, Seoul 02455, Republic of Korea
}

(Received 5 November 2017; published 28 February 2018)

\begin{abstract}
In this paper, we discuss and calculate the electroweak parameters $R_{l}, A_{l}$, and $N_{\nu}^{l}$ in a model that combines an inverse seesaw with the scotogenic model. Dark matter relic density is also considered. Because of the stringent constraint from the ATLAS experimental data, it is difficult to detect the loop effect on $R_{l}, A_{l}$ in this model considering both the theoretical and the future experimental uncertainties. However, $N_{\nu}^{l}$ can sometimes become large enough for the future experiments to verify.
\end{abstract}

DOI: 10.1103/PhysRevD.97.035020

\section{INTRODUCTION}

The type I seesaw mechanisms [1-5] are utilized to explain the smallness of the neutrino masses by introducing some extremely heavy right-handed neutrinos with the masses $10^{8-12} \mathrm{TeV}$, which is far beyond the ability of any current or proposed collider facility. Suppressing the mass scales of the right-handed neutrinos below $1 \mathrm{TeV}$ will also lead to tiny Yukawa couplings $\left(\sim 10^{(-7)-(-9)}\right)$, making it rather difficult to produce any experimental signals in reality.

The scotogenic model [6-9] and the inverse seesaw model [10-13] are the two different approaches toward the $\mathrm{TeV}$-scale phenomenology corresponding to the neutrino sector. In the various versions of the scotogenic model, the active neutrinos acquire masses through loop corrections. In this case, the loop factor naturally suppresses the Majorana masses of the left-handed neutrinos. As for the inverse seesaw model, two groups of so-called "pseudoDirac" sterile neutrinos are introduced. The contributions from the large Yukawa couplings to the left-handed neutrino masses are nearly canceled out, with a small remnant left over due to the small Majorana masses among the pseudo-Dirac sterile neutrinos which softly break the lepton number.

As far as we know about the literature, the combination of these two models can date back to Ref. [14], which appeared shortly after Ref. [6]. There are also various papers in the literature suggesting different variants or discussing the phenomenologies. (For some examples, see Refs. [15-18], while Ref. [19] had discussed a similar

\footnotetext{
*tangyilei@kias.re.kr
}

Published by the American Physical Society under the terms of the Creative Commons Attribution 4.0 International license. Further distribution of this work must maintain attribution to the author(s) and the published article's title, journal citation, and DOI. Funded by SCOAP ${ }^{3}$. linear seesaw model.) In this paper, we discuss a simple version of this type of models motivated by avoiding some tight restrictions on the Yukawa coupling orders. In the usual scotogenic models, Yukawa couplings are usually constrained by the leptonic flavor changing neutral current (FCNC) such as the $\mu \rightarrow e \gamma$ bound. In the case of the inverse seesaw mechanisms, the invisible decay width of the $Z$ boson also exerts limits on the Yukawa couplings. This leads to the mixings between the active neutrinos and the sterile neutrinos and will result in the corrections to the $Z \rightarrow \nu \nu$ branching ratios on the tree level. Combining these two models can reach some relatively larger Yukawa couplings, while evading some constraints at the same time.

\section{MODEL DESCRIPTIONS}

The scotogenic model is based on the inert two Higgs doublet model (ITHDM). In this model, two $S U(2)_{L}$ Higgs doublets $\Phi_{1}$ and $\Phi_{2}$ are introduced. Let $\Phi_{2}$ be $Z_{2}$ odd, while $\Phi_{1}$ together with other standard model (SM) fields are $Z_{2}$ even, and the potential for the Higgs sector is given by

$$
\begin{aligned}
V= & m_{1}^{2} \Phi_{1}^{\dagger} \Phi_{1}+m_{2}^{2} \Phi_{2}^{\dagger} \Phi_{2}+\frac{\lambda_{1}}{2}\left(\Phi_{1}^{\dagger} \Phi_{1}\right)^{2}+\frac{\lambda_{2}}{2}\left(\Phi_{2}^{\dagger} \Phi_{2}\right)^{2} \\
& +\lambda_{3}\left(\Phi_{1}^{\dagger} \Phi_{1}\right)\left(\Phi_{2}^{\dagger} \Phi_{2}\right)+\lambda_{4}\left(\Phi_{1}^{\dagger} \Phi_{2}\right)\left(\Phi_{2}^{\dagger} \Phi_{1}\right) \\
& +\frac{\lambda_{5}}{2}\left[\left(\Phi_{1}^{\dagger} \Phi_{2}\right)^{2}+\left(\Phi_{2}^{\dagger} \Phi_{1}\right)^{2}\right],
\end{aligned}
$$

where $\Phi_{1,2}$ are the two Higgs doublets with the hypercharge $Y=\frac{1}{2}, \lambda_{1-5}$ are the coupling constants, and $m_{1}^{2}, m_{2}^{2}$ are the mass parameters.

In the ITHDM, only $\Phi_{1}$ acquires the electroweak vacuum expectation value $v$, and the SM Higgs $h$ originates from this doublet. All the elements of the $\Phi_{2}$ form the other scalar bosons $H^{ \pm}, H, A$, and no mixing between the SM Higgs and the exotic bosons takes place. Therefore, 


$$
\Phi_{1}=\left(\begin{array}{c}
G^{+} \\
\frac{v+h+i G^{0}}{\sqrt{2}}
\end{array}\right), \quad \Phi_{2}=\left(\begin{array}{c}
H^{+} \\
\frac{H+i A}{\sqrt{2}}
\end{array}\right)
$$

Because of the $Z_{2}$ symmetry, all the fermions $Q_{L}, u_{R}, d_{R}$, $L_{L}, e_{R}$ only couple with the $\Phi_{1}$ field

$$
\begin{aligned}
\mathcal{L}_{\text {Yukawa }}^{\mathrm{SM}}= & -Y_{u i j} \bar{Q}_{L i} \tilde{\Phi}_{1} u_{R j}-Y_{d i j} \bar{Q}_{L i} \Phi_{1} d_{R j} \\
& -Y_{l i j} \bar{L}_{L i} \Phi_{1} l_{R j}+\text { H.c. },
\end{aligned}
$$

where $Y_{u, d, l}$ are the $3 \times 3$ coupling constants.

The $Z_{2}$-odd pseudo-Dirac sterile neutrinos $N_{i}=P_{L} N_{L i}+$ $P_{R} N_{R i}\left(i=1-3, P_{L, R}=\frac{1 \mp \gamma^{5}}{2}\right)$ together with the left-handed lepton doublets couple with the $\Phi_{1}$. The pseudo-Dirac 4-spinors $N_{i}$ can be written in the form of $\left[\begin{array}{c}N_{L i}^{\mathrm{w}} \\ i \sigma^{2} N_{R_{i}}^{\mathrm{W} *}\end{array}\right]$, where $N_{\mathrm{L}, \mathrm{R} i}^{\mathrm{w}}$ are the sterile neutrino fields in the Weyl 2-spinor form. The corresponding Lagrangian is given by

$$
\begin{aligned}
\mathcal{L}_{\text {Yukawa,Mass }}^{\nu}= & -Y_{N i j} \bar{L}_{L i} \tilde{\Phi}_{2} N_{R j}-m_{N i j} \bar{N}_{i} N_{j} \\
& -\mu_{i j}^{1} \overline{N_{R i}^{c}} N_{R j}-\mu_{i j}^{2} \overline{N_{L i}^{c}} N_{L j},
\end{aligned}
$$

where $Y_{N}$ is the $3 \times 3$ Yukawa coupling constant matrix, $m_{N}$ is the $3 \times 3$ Dirac mass matrix between the sterile neutrino pairs, $\mu$ is a $3 \times 3$ mass matrix that softly breaks the lepton number, and $N_{L, R i}^{c}=-i \gamma^{2} \gamma^{0}{\overline{N_{L, R i}^{c}}}^{T}$ is the charge conjugate transformation of the $N_{L, R i}$ field. However, as for the tree-level inverse seesaw model, there exist examples in which only the mass terms corresponding to $\mu_{i j}^{2} \overline{N_{L i}^{c}} N_{L j}$ are generated and discussed [20-22]. In fact, it is easier to generate the correct light neutrino mass matrix pattern in a discrete symmetry and flavon-based model if the lepton flavor violation has only one source (it, in this paper, refers to $\overline{N_{L i}^{c}} N_{L j}$ ), though, in this paper, we discuss both the contributions from $\mu^{1,2}$ for completion.

\section{NEUTRINO MASSES}

The right panel of Fig. 1 shows the diagram that induces the neutrino masses. Inside the loop there is a Majonara mass insertion term originated from Eq. (4). By principle, we can directly calculate through this diagram; however, in this paper, we adopt another method. In fact, the

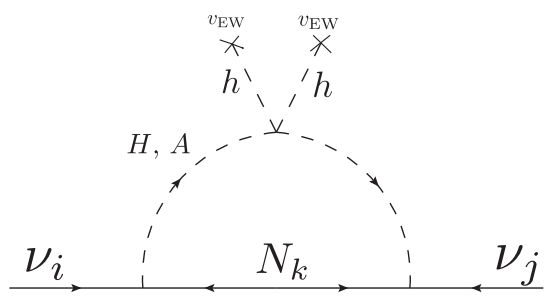

pseudo-Dirac neutrinos can actually be regarded as a pair of nearly degenerate Majorana fermions. Each fermion contributes to the left panel of Fig 1, and by summing over all the results, a remnant proportional to the $\mu_{i j}$ is left over.

In spite of the coupling constants, the kernel of the left panel of Fig. 1 is given by Ref. [6],

$f\left(M_{k}, m_{H}, m_{A}\right)=\frac{M_{k}}{16 \pi^{2}}\left[\frac{m_{H}^{2}}{m_{H}^{2}-M_{k}^{2}} \ln \frac{m_{H}^{2}}{M_{k}^{2}}-\frac{m_{A}^{2}}{m_{A}^{2}-M_{k}^{2}} \ln \frac{m_{A}^{2}}{M_{k}^{2}}\right]$,

where $M_{k}$ and $m_{H, A}$ are the mass of the Majorana sterile neutrino and the masses of the $C P$-even and $C P$-odd neutral exotic Higgs bosons $H$ and $A$.

In the Weyl basis, the mass terms in (4) can be written in the form of

$\mathcal{L}_{\text {Mass }}^{\mathrm{SM}}=m_{N_{i j}}\left(N_{L i}^{\mathrm{w}} N_{R j}^{\mathrm{w}}+\right.$ H.c. $)+\mu_{i j}^{1} N_{R i}^{\mathrm{w}} N_{R j}^{\mathrm{w}}+\mu_{i j}^{2} N_{L i}^{\mathrm{w}} N_{L j}^{\mathrm{w}}$.

That is to say, in the $N_{L i}, N_{R i}$ basis, the blocking mass matrix is given by

$$
M_{N}=\left[\begin{array}{cc}
\mu^{1} & m_{N} \\
m_{N}^{T} & \mu^{2}
\end{array}\right]
$$

where $m_{N}=\left[m_{N i j}\right]$ and $\mu^{1,2}=\left[\mu_{i j}^{1,2}\right]$ are the $3 \times 3$ submatrix. Without loss of generality, let $m_{N}$ be diagonalized with the eigenvalue $m_{N i}, i=1,2,3$, regard $\mu_{i j}^{1,2}$ as the perturbation parameter, and diagonalize (7), so the rotation matrix is given by

$$
\begin{aligned}
M_{N} \Rightarrow V^{T} M_{N} V, \quad V & =\frac{1}{\sqrt{2}}\left[\begin{array}{cc}
I & I \\
-I & I
\end{array}\right] \delta, \\
\delta & =\left[\begin{array}{cc}
I_{n} & C \\
-C^{T} & -I_{n}
\end{array}\right],
\end{aligned}
$$

where

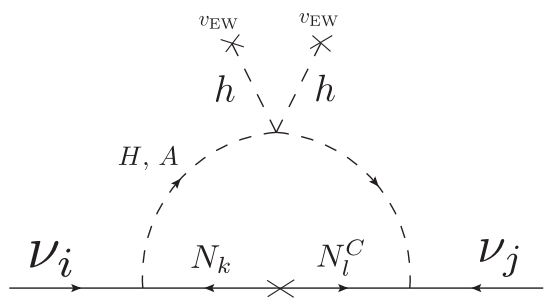

FIG. 1. The left panel shows the neutrino loop-induced mass in the case of Majorana sterile neutrinos. The right panel shows the case of pseudo-Dirac sterile neutrinos. 


$$
\begin{aligned}
I_{n i j} & =\frac{\mu_{i j}^{-}}{2\left(m_{N j}-m_{N i}\right)} \text { for } i \neq j, \quad I_{n i j}=0 \text { for } i=j, \\
C_{i j} & =\frac{\mu_{i j}^{+}}{2\left(-m_{N j}-m_{N i}\right)},
\end{aligned}
$$

where $\mu_{i j}^{+}=\mu_{i j}^{1}+\mu_{i j}^{2}$ and $\mu_{i j}^{-}=\mu_{i j}^{2}-\mu_{i j}^{1}$. Replacing each mass in (5) with $\left(V^{T} M_{N} V\right)_{i i}$, multiplying the coupling constants $Y_{N i k} Y_{N j l}$, and then summing over all the terms while dropping higher orders of $\mu_{i j}$, we acquire

$$
\begin{aligned}
m_{\nu i j}= & \sum_{k, l=1-3} Y_{N i k} Y_{N j l}\left[\mu_{k l}^{-} F\left(m_{N l}, m_{N k}, m_{H}, m_{A}\right)\right. \\
& \left.-\mu_{k l}^{+} \frac{f\left(m_{N l}, m_{H}, m_{A}\right)+f\left(m_{N k}, m_{H}, m_{A}\right)}{m_{N l}+m_{N k}}\right],
\end{aligned}
$$

where

$$
\begin{aligned}
& F\left(m_{N l}, m_{N k}, m_{H}, m_{A}\right) \\
& \quad=\left\{\begin{array}{cl}
\frac{f\left(m_{N l}, m_{H}, m_{A}\right)-f\left(m_{N k}, m_{H}, m_{A}\right)}{m_{N l}-m_{N k},} & \text { when } m_{N l} \neq m_{N k}, \\
\frac{\partial f\left(m_{N l}, m_{H}, m_{A}\right)}{\partial m_{N l}}, & \text { when } m_{N l}=m_{N k} .
\end{array}\right.
\end{aligned}
$$

In this paper, we ignore all the $C P$ phases for simplicity and adopt the central values $[23,24]$

$$
\begin{aligned}
\Delta m_{21}^{2} & =7.37 \mathrm{eV}^{2}, \quad\left|\Delta m^{2}\right|=\left|\Delta m_{32}^{2}+\Delta \frac{m_{21}^{2}}{2}\right| \\
& =2.50 \mathrm{eV}^{2}, \quad \sin \theta_{12}^{2}=0.297, \\
\sin ^{2} \theta_{23} & =0.437, \quad \sin ^{2} \theta_{13}=0.0214
\end{aligned}
$$

to calculate the $m_{\nu}$ through the Pontecorvo-MakiNakagawa-Sakata matrix

$$
\begin{aligned}
U & =\left[\begin{array}{ccc}
c_{12} c_{13} & s_{12} c_{13} & s_{13} e^{-i \delta} \\
-s_{12} c_{23}-c_{12} s_{23} s_{13} e^{i \delta} & c_{12} c_{23}-s_{12} s_{23} s_{13} e^{i \delta} & s_{23} c_{13} \\
s_{12} s_{23}-c_{12} c_{23} s_{13} e^{i \delta} & -c_{12} s_{23}-s_{12} c_{23} s_{13} e^{i \delta} & c_{23} c_{13}
\end{array}\right] \times \operatorname{diag}\left(1, e^{i \frac{\alpha_{21}}{2}}, e^{i \frac{\alpha_{31}}{2}}\right), \\
\operatorname{diag}\left(m_{1}, m_{2}, m_{3}\right) & =U^{T} m_{\nu} U,
\end{aligned}
$$

where $s_{i j}=\sin \theta_{i j}, c_{i j}=\cos \theta_{i j}$, and $\theta_{i j}$ 's are the mixing angles. The $C P$-phase angle $\delta$, and the two Majorana $C P$ phases $\alpha_{21,31}$ are omitted. $m_{1,2,3}$ are the masses of the three light neutrinos. Currently, the mass hierarchy (normal or inverse hierarchy) and the absolute neutrino masses still remain unknown. By assuming the mass hierarchy and the lightest neutrino mass $m_{\nu 0}$, matrix $m_{\nu}$ can be computed and then $\mu$ can be calculated through inversely solving Eq. (10).

\section{DISCUSSIONS ON OBLIQUE PARAMETERS, $l_{1} \rightarrow l_{2} \gamma$, AND THE COLLIDER CONSTRAINTS}

The one-loop level contributions to the Peskin-Takeuchi oblique parameters $S, T$, and $U$ from the general two Higgs doublet model (THDM) have been calculated in the literature $[25,26]$. Some papers (e.g., Refs. $[27,28])$ also plot the allowed region constrained by the oblique parameters. From the formula and the figures in the literature, we can easily find that if $m_{A} \approx m_{H^{ \pm}}$, or if $m_{H} \approx m_{H^{ \pm}}$, the contributions from the exotic Higgs doublets will nearly disappear in the alignment limit. Therefore, in this paper, we discuss the following benchmark parameter spaces:

(i) $m_{H}=m_{H^{ \pm}}, m_{A} \leq m_{H^{ \pm}}$,

(ii) $m_{A}=m_{H^{ \pm}}, m_{H} \leq m_{H^{ \pm}}$.

Although $m_{H, A}>m_{H^{ \pm}}$is also possible, however, this will make some parameters decouple, and we aim at discussing as much phenomenology (allowed by the current constraints) as possible in this paper. In this paper, we do not discuss such parameter space.

The leptonic FCNC decays $l_{1} \rightarrow l_{2}+\gamma$ in Fig. 2 set constraints on the parameter space. In the original scotogenic model, Ref. [7] had pointed out that in the usual scotogenic model, the parameter space is quite constrained by the $\mu \rightarrow e \gamma$ bounds. The nearly degenerate neutrino mass scenario to avoid this bound has become very

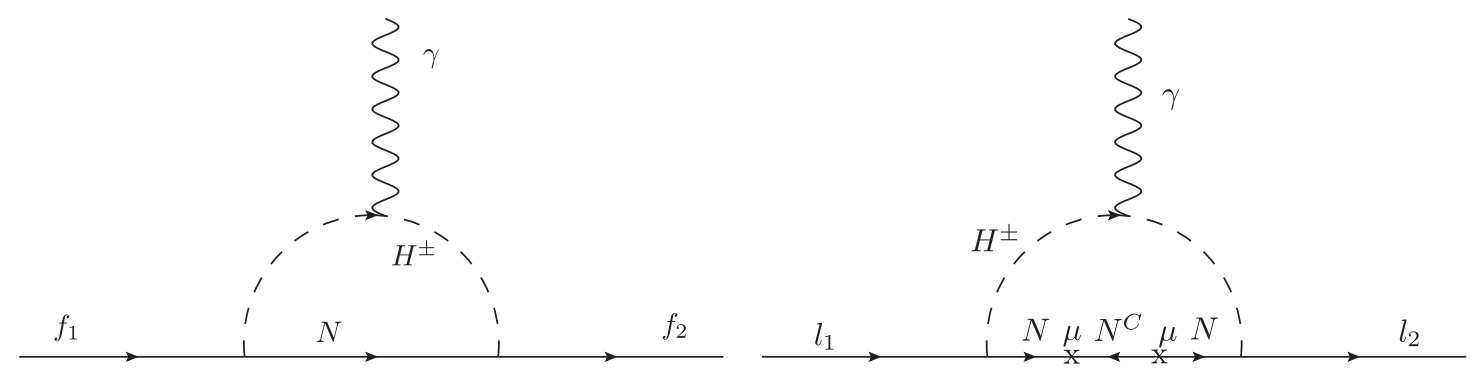

FIG. 2. The $l_{1} \rightarrow l_{2} \gamma$ diagrams. 
unfavorable considering the recent cosmological bound on the neutrino masses [29] together with the oscillation data. Similar to the cases in Ref. [30], the FCNC elements in the $Y_{N i j}$ and the $m_{N i j}$ are stringently bounded through the diagram in the left panel of Fig. 2. Setting $m_{N} \propto I$ and $Y_{N i j} \propto I$ will simply avoid this problem, where $I$ is the identity matrix. This is not ad hoc, if some flavon-based inverse-seesaw models such as those in Refs. [20-22] that describe the origin of these parameters are transplanted to our loop-level case. Eventually, if all of the leptonic FCNC effects originate from the $\mu$ terms, the diagram in the right panel of Fig. 2 will become the lowest order of contributions to $l_{1} \rightarrow l_{2}+\gamma$ and will become severely suppressed by the factor $\sim \frac{\mu^{4}}{m_{N}^{4}}$. Therefore, in this paper, we only consider the case that $m_{N} \propto I, Y_{N i j} \propto I$, and $\mu \propto I$.

In this paper, we are also interested in the case that the fermionic $N$ 's are the lightest $Z_{2}$-odd particles that turn out to be the candidate of the dark matter. $H$ and $A$ are not considered partly because such cases have been widely and sufficiently talked about in the literature.

On the collider, usually $H^{ \pm}, H$, and $A$ are produced by the electroweak processes and then decay into the missing energy plus some SM final states. Notice that the decay rate between different sterile neutrino $N_{i}$ 's are so severely suppressed by the smallness of their mass differences due to $m_{N} \propto I$ and $\mu \ll m_{N}$ that we assume such decay will never happen inside the detector. In fact, even if they can decay inside the detector, this will only produce some rather soft objects that might be difficult to figure out. Here we regard all the $N_{i}$ 's as the missing energies (ME), and we have examined various combinations of the production processes $p p \rightarrow H^{+} H^{-}, p p \rightarrow H^{ \pm} H, p p \rightarrow H^{ \pm} A, p p \rightarrow H A$ with the various decay channels $H^{ \pm} \rightarrow N_{i} l_{j}^{ \pm}, H^{ \pm} \rightarrow A / H+W^{ \pm}$,

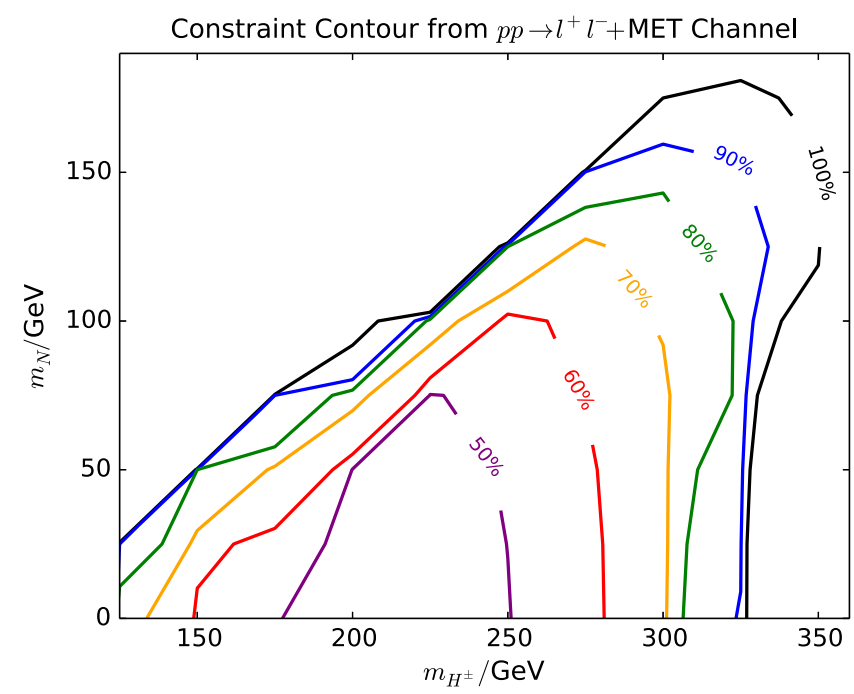

FIG. 3. The $95 \%$ C.L. exclusion limits on the $m_{H^{ \pm}}-m_{N}$ parameter space. The results on different branching ratios of $H^{ \pm} \rightarrow N_{i} l_{j}^{ \pm}$(printed on each curve in the panel) are plotted.
$H(A) \rightarrow A(H)+Z$, etc. The LHC experiments have extracted the data on some of the channels, and among these we select the most stringent one, which is $p p \rightarrow H^{+} H^{-} \rightarrow l^{+} l^{-}+\mathrm{ME}$. For this final state, the ATLAS Collaboration has provided the binned data of the SM background in Ref. [31]. Here, we implement the model files and generate the events by FeynRules 2.3.28 [32] + MadGraph 2.5.5 [33], and bin our results by Madanalysis 5.1.5 [34-36]. Both the same flavor and the different flavor data are considered. Since the final states

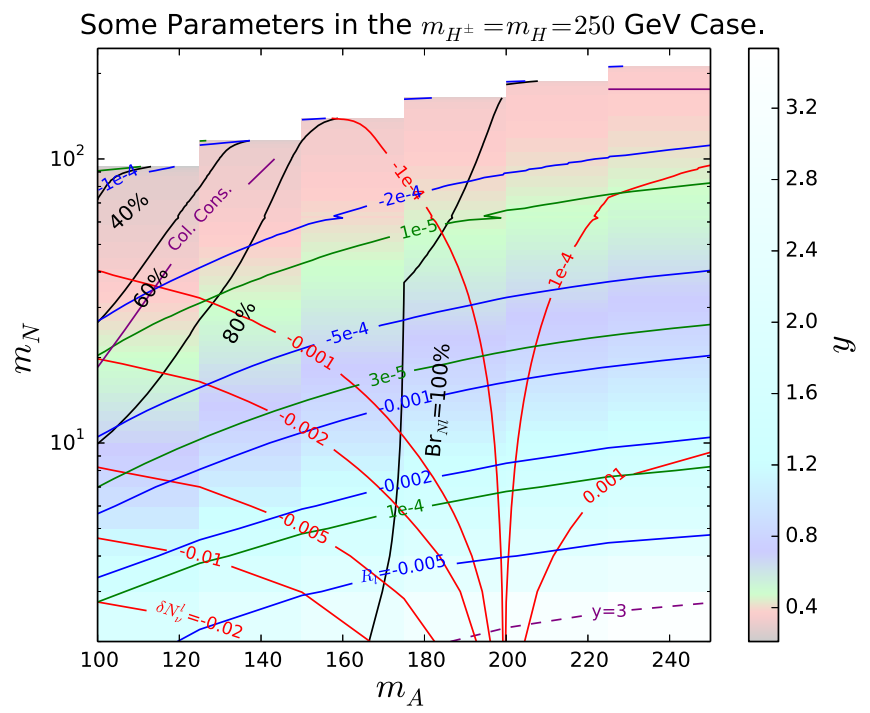

Some Parameters in the $m_{H^{ \pm}}=m_{A}=250 \mathrm{GeV}$ Case.

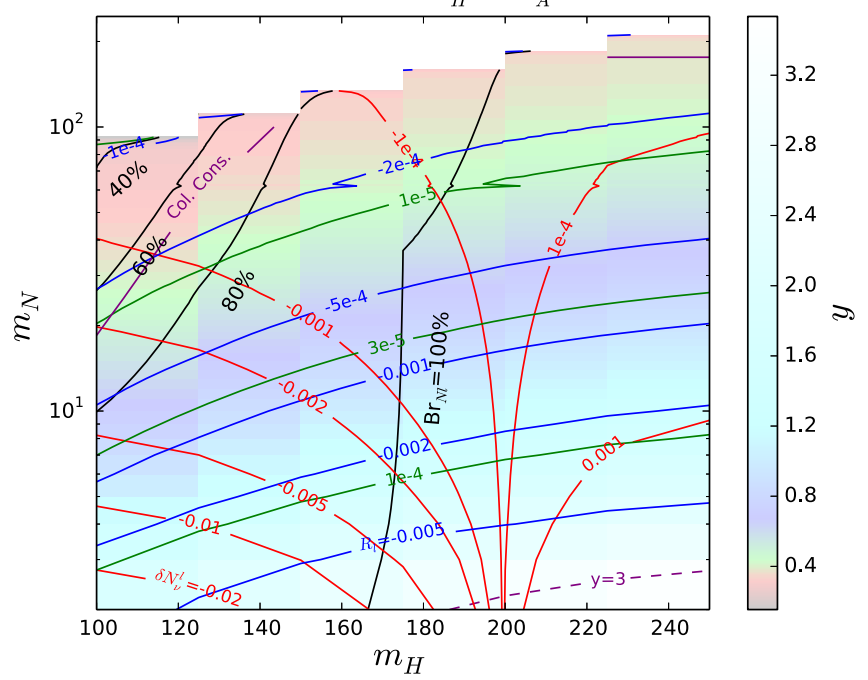

FIG. 4. $\delta R_{l}$ (blue lines), $\delta A_{l}$ (green lines), and $\delta N_{\nu}^{l}$ (red lines) in the $m_{H^{ \pm}}=250 \mathrm{GeV}$ case. The colors in the background indicate the Yukawa coupling $y$ that can result in the correct relic density. The dashed purple line in the bottom right of the picture shows the $y=3 \sim \sqrt{4 \pi}$ boundary of the Yukawa coupling. The black lines indicate the branching ratio of the $H^{ \pm} \rightarrow N_{i} l_{j}^{ \pm}$decay channel, and the solid purple line marked with "Col. Cons." gives the C.L. $95 \%$ collider constraint derived from Fig. 3. The right-down direction from this line has been excluded. 

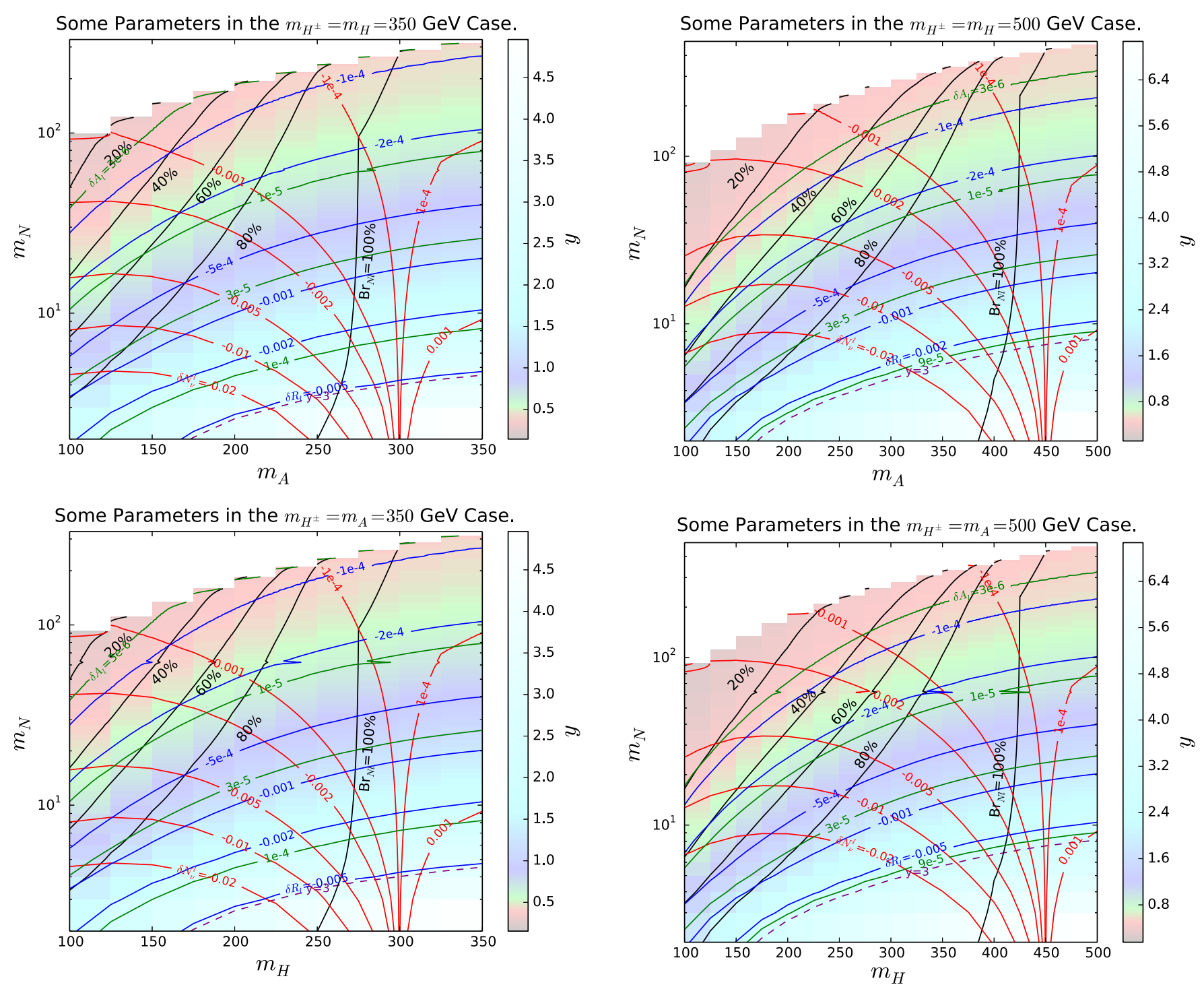

FIG. 5. Results in the $m_{H^{ \pm}}=350 \mathrm{GeV}$ case. The meanings of the colors and the lines are similar to those in Fig. 4.

are leptons, only parton-level analyses are processed. We have calculated the C.L. ratio according to Ref. [37] and scanned in the $m_{H^{ \pm}}-m_{N}$ parameter space. We plot our results of the 95\% C.L. exclusion limits in Fig. 3.

\section{CALCULATIONS OF SOME OBSERVABLES}

In this section, we aim at calculating the following observables:

(i) Relic density of the dark matter.

(ii) Shiftings on the $Z$-resonance observables $R_{l}=\frac{\Gamma_{Z \rightarrow \text { hadrons }}}{\Gamma_{Z \rightarrow l^{+} l^{-}}}$, $A_{l}=\frac{2 \bar{g}_{\bar{g}}^{l} \bar{g}_{A}^{l}}{\bar{g}_{V}^{2}+\bar{g}_{A}^{2}}$.

(iii) Shiftings on the $Z \rightarrow$ invisible parameter $N_{\nu}^{l}=$ $\frac{\Gamma_{\text {inv }}^{Z}}{\Gamma_{l}^{Z}}\left(\frac{\Gamma_{l}^{Z}}{\Gamma_{\nu}^{Z}} \mathrm{SM}^{\cdot}\right.$

The review on $R_{l}, A_{l}$, and $N_{\nu}^{l}$ can be found in Ref. [24]. The relic density of the dark matter is calculated by

FIG. 6. Results in the $m_{H^{ \pm}}=500 \mathrm{GeV}$ case. The meanings of the colors and the lines are similar to those in Fig. 4.

micrOMEGAs 4.3.5 [38,39], with our model file exported by FeynRules 2.3.28. The inert THDM part of the model file is based on Refs. [40,41].

The shiftings on the electroweak parameters $\delta R_{l}, \delta A_{l}$, and $\delta N_{\nu}^{l}$ are calculated according to the formulas and steps listed in Ref. [30], where the one-loop corrections to the $Z-l-l$ coupling constants are computed and then their values are replaced by the expressions of $\delta R_{l}, \delta A_{l}$, and $\delta N_{\nu}^{l}$, which depend on them. The computing processes can be compared and checked with Ref. [42]. Note that in the case of this paper, neither the tree-level correction to the muon's decay constant $G_{F}$ nor the tree-level mixing between the sterile neutrinos and the light neutrinos exists; therefore the computing procedures are much simpler than those in Ref. [30].

To present our results, we only consider the subparameter space of $m_{H^{ \pm}}=250,350,500 \mathrm{GeV}$. The Yukawa 
coupling constant $\left[Y_{N i j}\right]=y I$ is adjusted in order for the relic density $\Omega_{\mathrm{DM}} h^{2}$ to approach $0.1199 \pm 0.0027$ [43]. Combined with the two cases in the last section, we show six plots in Figs. 4, 5, and 6.

As has been mentioned, we are only interested in the case when $m_{N}<m_{H, A}$; therefore the upper-left part of the plots are all left blank. The color boundary becomes a steplike shape due to the insufficient density of points on the horizontal axis and our limit on the computational resources.

\section{DISCUSSIONS}

There are plans for future experiments to measure $R_{l}, A_{l}$, and $N_{\nu}^{l}$ [44-47]. Currently, the collider experiments have imposed very stringent bounds on the mass of $m_{H}^{ \pm}$. From Fig. 3, we can easily see that $m_{H}^{ \pm} \lesssim 325 \mathrm{GeV}$ has been excluded in the case when $m_{N} \rightarrow 0$ and $\mathrm{Br}_{H^{ \pm} \rightarrow N l^{ \pm}}=100 \%$. When $\mathrm{Br}_{H^{ \pm} \rightarrow N l^{ \pm}}<100 \%$, bounds on $m_{H^{ \pm}}$can somehow be relaxed. However, besides the leptonic channel, $H^{ \pm}$can only decay into $H / A+W^{ \pm}$, which requires $m_{H^{ \pm}}-m_{H / A} \gtrsim$ $100 \mathrm{GeV}$. This substantially compresses the unconstrained parameter space in the case when $m_{H}^{ \pm} \lesssim 325$. As an example, it is obvious that in Fig. 4, only a fraction in the upper-left part of the plot remains unconstrained.

As for $\delta R_{l}, \delta A_{l}$, all the corresponding diagrams only involve $H^{ \pm}$, Thus they are highly suppressed due to the constraints on $m_{H^{ \pm}}$. As has been discussed in Ref. [30], taking both the experimental and the theoretical uncertainties into account, $\left|\delta R_{l}\right|$ should be $\gtrsim 0.001$ and $\left|\delta A_{l}\right|$ should be $\gtrsim 8 \times 10^{-5}$ in order for the new physics effects to be observed in future experiments that measure the electroweak parameters. When $m_{H^{ \pm}}>325 \mathrm{GeV}$, for example, as in Figs. 5 and 6, in some cases the predicted $\left|\delta R_{l}\right|$ or $\left|\delta A_{l}\right|$ might reluctantly reach this bound; however, in this case the Yukawa coupling $y$ approaches the perturbative constraint $y<\sqrt{4 \pi} \sim 3$. On the other hand, when $m_{H^{ \pm}} \lesssim 350 \mathrm{GeV}$, for example, when $m_{H^{ \pm}}=250 \mathrm{GeV}$ as shown in Fig. 4, the unconstrained parameter space usually refers to a too-small Yukawa coupling for $\left|\delta R_{l}\right|$ or $\left|\delta A_{l}\right|$. Therefore, it is difficult to detect the effects on $\delta R_{l}$ and $\delta A_{l}$ from this model on future experiments.

However, $\delta N_{\nu}^{l}$ can be large if $m_{H}$ or $m_{A}$ is relatively small. Reference [47] has shown us that $\delta N_{\nu}^{l}$ can reach a statistical uncertainty of 0.00004 and a systematic uncertainty of 0.004 in its Table 1. However, the discussions in Sec. VII [47] mentioned that a desirable goal would be to reduce this uncertainty to 0.001. In this case, the Future Circular Collider with electron-positron beams is enough to cover much of the parameter space in $m_{H^{ \pm}}=350$ and $500 \mathrm{GeV}$. However, as has been shown in Fig. 4 , it is still difficult to detect $\delta N_{\nu}^{l}$ in its unconstrained parameter space when $m_{H^{ \pm}}=250 \mathrm{GeV}$. Interestingly, current LEP results $N_{\nu}^{l}=2.984 \pm 0.008$ show a $2 \sigma$ deviation from the standard model prediction. If this will be confirmed in future collider experiments, it will become circumstantial evidence to this model.
Finally, we should discuss the direct detection on this model. Since we have only talked about the case of the sterile neutrino playing the role of dark matter, there is no tree-level diagram for the nucleon-dark matter interactions. Reference [48] has calculated the loop-induced $Z$-portal cross sections for the nucleon-dark matter collisions in both the Majorana and the Dirac cases. In our paper, the dark matter particle is the lightest Majorana mass eigenstate of a group of pseudo-Dirac fermions. The mass difference $\delta$ between the two Majorana elements of one pseudo-Dirac pair of fermions is controlled by $\mu$. According to (10), $\mu$ is inversely proportional to $F\left(m_{N l}, m_{N k}, m_{H}, m_{A}\right)-\frac{f\left(m_{N_{l}}, m_{H}, m_{A}\right)+f\left(m_{N_{k}}, m_{H}, m_{A}\right)}{m_{N_{l}}+m_{N_{k}}}$, and $f\left(m_{N_{l, k}}, m_{H}, m_{A}\right), F\left(m_{N_{l}}, m_{N_{k}}, m_{H}, m_{A}\right)$ approaches zero in the limit $m_{N} \rightarrow 0$ or $m_{A} \rightarrow m_{H}$. Generally speaking, $\mu \sim 100 \mathrm{eV}$ if no particular pattern of values are appointed to $m_{H, A, N}$. For example, if the lightest left-handed neutrino is $m_{\nu 0}=0.03 \mathrm{eV}$ in the case of the normal mass hierarchy, and we assign $m_{H}=350 \mathrm{GeV}, m_{A}=200 \mathrm{GeV}$, $m_{N}=100 \mathrm{GeV}, y=0.447$, therefore $\mu_{i i} \simeq 100 \mathrm{eV}$ and $\mu_{i j} \simeq 10 \mathrm{eV}(i \neq j)$. However, if $m_{A} \simeq m_{H}, \mu$ will be substantially amplified. For example, when $m_{H}=350 \mathrm{GeV}$, $m_{A}=349.5 \mathrm{GeV}, \quad m_{N}=100 \mathrm{GeV}, y=0.605$, in this case $\mu \simeq 10 \mathrm{keV}$. Furthermore, if we again appoint $m_{N}=10 \mathrm{GeV}$, and let $y=1.88$ in order for a correct relic density, $\mu$ will become $\simeq 100 \mathrm{keV}$. Therefore, in this model, the mass splitting $\delta$ can be large enough ( $\gtrsim 100 \mathrm{keV}$ ) so that the dark matter can be regarded as a pure Majorana particle in some parameter space, while in some parameter space, the mass difference $\delta$ might become so small $(\sim 100 \mathrm{eV})$ so that the dark matter can transfer between the two mass eigenstates during the collision with the nucleons. The latter case is more similar to the Dirac case discussed in Ref. [48]. According to [48], the Z-portal spin-dependent cross section was calculated to be less than $10^{-41} \mathrm{~cm}^{2}$ in both the Dirac case and the Majorana case, while the spin-independent cross section in the Dirac case was calculated to be $\lesssim 10^{-47} \mathrm{~cm}^{2}$ when $m_{N}<200 \mathrm{GeV}$. Although these are still below the experimental bounds [49-52], it is hoped that future experiments will cover some of our parameter space since the current bounds are not far from the predictions.

\section{CONCLUSIONS}

We have discussed some phenomenologies of a simple inverse seesaw scotogenic model by calculating the electroweak parameters $R_{l}, A_{l}$, and $N_{\nu}^{l}$ in the case of a correct dark matter relic density. The current ATLAS results have imposed stringent bounds on the parameter space, lowering the predicted $R_{l}$ and $A_{l}$. Considering both the experimental and the theoretical uncertainties, it is difficult to detect the effect from this model on $R_{l}$ and $A_{l}$ in future measurements. However, $\delta N_{\nu}^{l}$ can become large enough, shedding light on verifying or constraining this model in the future. 


\section{ACKNOWLEDGMENTS}

We thank Ran Ding, Pyungwon Ko, and Peiwen Wu for helpful discussions. This work was supported by the Korea Research Fellowship Program through the National Research Foundation of Korea (NRF) funded by the Ministry of Science and ICT (2017H1D3A1A01014127), and it is also supported in part by National Research Foundation of Korea (NRF) Research Grant No. NRF2015R1A2A1A05001869. We also thank the Korea Institute for Advanced Study for providing computing resources (KIAS Center for Advanced Computation Abacus System) for this work.
[1] P. Minkowski, Phys. Lett. 67B, 421 (1977).

[2] T. Yanagida, in Proceedings of the Workshop on Unified Theory and Baryon Number of the Universe (KEK, Tsukuba, 1979), p. 95

[3] P. R. M. Gell-Mann and R. Slansky, Report No. CALT-68709, 1979; in Supergravity (North-Holland, Amsterdam, 1979), p. 315.

[4] S. Glashow, in Quarks and Leptons (Plenum, New York, 1980), p. 707.

[5] R. N. Mohapatra and G. Senjanovic, Phys. Rev. Lett. 44, 912 (1980).

[6] E. Ma, Phys. Rev. D 73, 077301 (2006).

[7] J. Kubo, E. Ma, and D. Suematsu, Phys. Lett. B 642, 18 (2006).

[8] D. Suematsu, T. Toma, and T. Yoshida, Phys. Rev. D 79, 093004 (2009).

[9] S.-Y. Ho and J. Tandean, Phys. Rev. D 87, 095015 (2013).

[10] D. Wyler and L. Wolfenstein, Nucl. Phys. B218, 205 (1983).

[11] R. N. Mohapatra and J. W. F. Valle, Phys. Rev. D 34, 1642 (1986).

[12] E. Ma, Phys. Lett. B 191, 287 (1987).

[13] R. N. Mohapatra, Phys. Rev. Lett. 56, 561 (1986).

[14] T. Hambye, K. Kannike, E. Ma, and M. Raidal, Phys. Rev. D 75, 095003 (2007).

[15] H. Okada and T. Toma, Phys. Rev. D 86, 033011 (2012).

[16] I. Baldes, N. F. Bell, K. Petraki, and R. R. Volkas, J. Cosmol. Astropart. Phys. 07 (2013) 029.

[17] G. Guo, X.-G. He, and G.-N. Li, J. High Energy Phys. 10 (2012) 044.

[18] A. Ahriche, S. M. Boucenna, and S. Nasri, Phys. Rev. D 93, 075036 (2016).

[19] A. Das, T. Nomura, H. Okada, and S. Roy, Phys. Rev. D 96, 075001 (2017).

[20] M. Hirsch, S. Morisi, and J. W. F. Valle, Phys. Lett. B 679, 454 (2009).

[21] B. Karmakar and A. Sil, Phys. Rev. D 96, 015007 (2017).

[22] E. Ma, Phys. Rev. D 80, 013013 (2009).

[23] F. Capozzi, E. Lisi, A. Marrone, D. Montanino, and A. Palazzo, Nucl. Phys. B908, 218 (2016).

[24] C. Patrignani et al. (Particle Data Group), Chin. Phys. C 40, 100001 (2016).

[25] H. E. Haber and D. O’Neil, Phys. Rev. D 83, 055017 (2011).

[26] G. Funk, D. O'Neil, and R. M. Winters, Int. J. Mod. Phys. A 27, 1250021 (2012).

[27] A. Celis, V. Ilisie, and A. Pich, J. High Energy Phys. 12 (2013) 095.

[28] B. Coleppa, F. Kling, and S. Su, J. High Energy Phys. 01 (2014) 161.
[29] P. A. R. Ade et al. (Planck Collaboration), Astron. Astrophys. 594, A13 (2016).

[30] Y.-L. Tang and S.-h. Zhu, Phys. Rev. D 96, 055022 (2017).

[31] ATLAS Collaboration, Report No. ATLAS-CONF-2017039, 2017.

[32] A. Alloul, N. D. Christensen, C. Degrande, C. Duhr, and B. Fuks, Comput. Phys. Commun. 185, 2250 (2014).

[33] J. Alwall, R. Frederix, S. Frixione, V. Hirschi, F. Maltoni, O. Mattelaer, H. S. Shao, T. Stelzer, P. Torrielli, and M. Zaro, J. High Energy Phys. 07 (2014) 079.

[34] E. Conte, B. Fuks, and G. Serret, Comput. Phys. Commun. 184, 222 (2013).

[35] E. Conte, B. Dumont, B. Fuks, and C. Wymant, Eur. Phys. J. C 74, 3103 (2014).

[36] B. Dumont, B. Fuks, S. Kraml, S. Bein, G. Chalons, E. Conte, S. Kulkarni, D. Sengupta, and C. Wymant, Eur. Phys. J. C 75, 56 (2015).

[37] G. Cowan, K. Cranmer, E. Gross, and O. Vitells, Eur. Phys. J. C 71, 1554 (2011); 73, 2501(E) (2013).

[38] G. Belanger, F. Boudjema, A. Pukhov, and A. Semenov, Comput. Phys. Commun. 185, 960 (2014).

[39] G. Bélanger, F. Boudjema, A. Pukhov, and A. Semenov, Comput. Phys. Commun. 192, 322 (2015).

[40] A. Goudelis, B. Herrmann, and O. Stål, J. High Energy Phys. 09 (2013) 106.

[41] G. Belanger, B. Dumont, A. Goudelis, B. Herrmann, S. Kraml, and D. Sengupta, Phys. Rev. D 91, 115011 (2015).

[42] H. E. Haber and H. E. Logan, Phys. Rev. D 62, 015011 (2000).

[43] P. A. R. Ade et al. (Planck Collaboration), Astron. Astrophys. 571, A16 (2014).

[44] C.-S. S. Group, IHEP-CEPC-DR-2015-01, Institute of High Energy Physics.

[45] H. Baer et al., arXiv:1306.6352.

[46] C. C. JianPing Ma, Sci. China Phys. Mech. Astron. 53, 1947 (2010).

[47] M. Dam, arXiv:1601.03849.

[48] Q.-H. Cao, E. Ma, and G. Shaughnessy, Phys. Lett. B 673, 152 (2009).

[49] X. Cui et al. (PandaX-II Collaboration), Phys. Rev. Lett. 119, 181302 (2017).

[50] D. S. Akerib et al. (LUX Collaboration), Phys. Rev. Lett. 116, 161302 (2016).

[51] D. S. Akerib et al. (LUX Collaboration), Phys. Rev. Lett. 118, 021303 (2017).

[52] E. Aprile et al. (XENON Collaboration), Phys. Rev. Lett. 119, 181301 (2017). 